\title{
Mechanism of Growth Inhibition of Prostate Cancer Xenografts by Valproic Acid
}

\author{
Abhinav Sidana, ${ }^{1}$ Muwen Wang, ${ }^{1}$ Shabana Shabbeer, ${ }^{2}$ Wasim H. Chowdhury, ${ }^{1}$ George Netto, ${ }^{3}$ \\ Shawn E. Lupold, ${ }^{1}$ Michael Carducci, ${ }^{2}$ and Ronald Rodriguez ${ }^{1}$ \\ ${ }^{1}$ James Buchanan Brady Urological Institute, Johns Hopkins University School of Medicine, Marburg 205A, 600 North Wolfe Street, \\ Baltimore, MD 21287, USA \\ ${ }^{2}$ Sidney Kimmel Comprehensive Cancer Center, Johns Hopkins University School of Medicine, Baltimore, MD, USA \\ ${ }^{3}$ Department of Pathology, Johns Hopkins University School of Medicine, Baltimore, MD, USA
}

Correspondence should be addressed to Ronald Rodriguez, rrodrig@jhmi.edu

Received 20 May 2012; Revised 16 July 2012; Accepted 17 July 2012

Academic Editor: Eric W. Lam

Copyright () 2012 Abhinav Sidana et al. This is an open access article distributed under the Creative Commons Attribution License, which permits unrestricted use, distribution, and reproduction in any medium, provided the original work is properly cited.

\begin{abstract}
Valproic Acid (VPA), a histone deacetylase inhibitor, has been demonstrated to cause a marked decrease in proliferation of prostate cancer $(\mathrm{PCa})$ cells in vitro and a significant reduction in tumor volume in vivo. The goal of this study is to better understand the VPA-induced growth inhibition in vivo, by studying expression of various markers in PCa xenografts. Methods. For in vitro experiments, PCa cells were treated with $0,0.6$, and $1.2 \mathrm{mM}$ VPA for 14 days. For in vivo models, experimental animals received $0.4 \%$ VPA in drinking water for 35 days. Tissue microarray was generated using cell pellets and excised xenografts. Results. VPA treatment causes cell cycle arrest in PCa cells in vivo, as determined by increase in p21 and p27 and decrease in cyclin D1 expression. Increased expression of cytokeratin 18 was also seen in xenografts. LNCaP xenografts in treated animals had reduced androgen receptor (AR) expression. While decreased proliferation was found in vitro, increase in apoptosis was found to be the reason for decreased tumor growth in vivo. Also, an anti-angiogenic effect was observed after VPA treatment. Conclusion. VPA inhibits tumor growth by multiple mechanisms including cell cycle arrest, induction of differentiation, and inhibition of growth of tumor vasculature.
\end{abstract}

\section{Introduction}

The steady-state level of core histone acetylation is modulated by a dynamic balance between histone acetyl transferases and histone deacetylases (HDACs). Histone acetylation results in relaxation of the chromatin structure, allowing access to transcription factors whereas deacetylation is associated with repressed transcription [1]. HDACs are found in an aberrantly active state in many cancerous cells and cause repression of cell cycle inhibitors. This epigenetic alteration of histones is an important posttranslational modification that regulates signaling pathways during neoplastic transformation [2]. The prospect of restoring the normal epigenetic state by pharmacologic targeting of these enzymes is thus a particularly promising anticancer strategy [2].

HDAC inhibitors (HDACIs) have been demonstrated in vitro to show antitumor activity by reexpression of repressed genes including cell cycle inhibitors. The cyclin-dependent kinase inhibitor (CDKI), p21 ${ }^{\text {WAFICIP1 }}$, which plays an important role in the arrest of cell growth, has been shown to be consistently induced by numerous HDACIs in in vitro studies $[3,4]$. The p21-related CDKI, p27 ${ }^{\mathrm{KIP} 1}$, also modulates the cell cycle and apoptosis, but its contribution to HDACI-induced processes remains to be fully elucidated [5].

Valproic Acid (VPA), an HDACI and an antiepileptic agent, inhibits class I and II HDACs [6]. In previous studies, we found that chronic administration of VPA results in a marked decrease in proliferation of prostate cancer cells in vitro and a significant reduction in tumor volume in vivo [79]. The goal of this study is to better understand the VPAinduced growth inhibition in vivo, by studying expression of markers of various cellular pathways in tissue microarrays (TMAs) generated from numerous human PCa cell xenografts in animals treated chronically with VPA. The 
study also serves to validate the in vitro findings of induction of cell cycle inhibitors in an in vivo model.

\section{Materials and Methods}

2.1. Tumor Cell Lines. Human prostate cancer cell lines LNCaP and DU145 were obtained from American Type Culture Collection (Manassas, VA), and C4-2 line was a gift from Dr. Leland Chung (Emory University, Atlanta, GA). All the cells were grown in RPMI 1640 with L-glutamine (Cellgro, Herndon, VA) supplemented with $10 \%$ heat-inactivated fetal bovine serum (FBS; Life Technologies, Inc., Carlsbad, CA), $5 \mu \mathrm{g} / \mathrm{mL}$ ciprofloxacin hydrochloride (US Biological, Swampscott, MA), and $50 \mu \mathrm{g} / \mathrm{mL}$ gentamicin (Quality Biological, Inc., Gaithersburg, MD). Cells were allowed to grow until $80 \%$ to $90 \%$ confluent and harvested with $0.05 \%$ trypsin/0.53 mmol/L EDTA (Cellgro, Herndon, VA) before each subsequent passage.

2.2. Establishment of Tumor Xenografts. Cell lines were grown to $80 \%$ to $90 \%$ confluence and harvested. Cells were resuspended in 1x phosphate-buffered saline ( $\mathrm{pH}$ 7.4; BioSource, Rockville, MD), mixed $1 \mathrm{x}$ with Matrigel (BD Biosciences, Palo Alto, CA), and injected $\left(1 \times 10^{6}\right.$ per injection $)$ subcutaneously into the lateral flanks of male athymic $n u / n u$ mice. Once palpable tumors were established, animals were randomized into control and treatment arms.

2.3. Valproic Acid Treatment. VPA ( $1 \mathrm{~mol} / \mathrm{L}$; VPA sodium salt; Sigma, St. Louis, MO) stock was made in PBS and filters sterilized through a $0.22 \mu \mathrm{m}$ filter. For in vitro experiments, cell lines were treated with $0,0.6$, and $1.2 \mathrm{mM}$ VPA for 14 days. Medium with freshly added VPA was changed every 48 hours. At the end of treatment cells were harvested with $0.05 \%$ trypsin/ $0.53 \mathrm{mmol} / \mathrm{L}$ EDTA (Cellgro, Herndon, VA) for generating cell pellets.

For in vivo experiments, animals received $0.4 \% \mathrm{w} / \mathrm{v}$ VPA in drinking water. This has been shown to produce blood levels in mouse [8] comparable to FDA-approved levels in humans [10]. Animals were treated for 35 days before excision of tumors.

2.4. TMAs: Construction and IHC Staining. For in vitro models, cells were harvested and washed in PBS. Resulting cell pellets were incubated for 1-2 hours in Bouin's fixative (75\% saturated picric acid, $20 \%$ formalin, $5 \%$ acetic acid), rinsed with 70\% ethanol and dehydrated according to standard procedures with ethanol and xylene. Cell pellets $>5 \mathrm{~mm}$ were split in order to achieve sufficient dehydration. Cells were embedded in paraffin following 90 min of incubation in liquid paraffin at $60^{\circ} \mathrm{C}$. For in vivo models, tumors were excised and portioned on day 35. Portions were fixed in buffered formalin and subsequently embedded in paraffin.

A tissue microarray (TMA) of the paraffin embedded materials was generated as described previously [11]. IHC stains for acetylated histone H3 (1:1000, Upstate, Charlottesville, VA), p21 ${ }^{\mathrm{WAF1} / \mathrm{CIP} 1}(1: 500$, Santa Cruz, Santa Cruz,CA), cyclin D1 (prediluted, Ventana Medical Systems, Tucson, AZ), p27 (1:100, DAKO, Carpinteria,CA), CK18
(1:100, DAKO, Carpinteria, CA), AR (1:250, Santa Cruz, Santa Cruz, CA), Ki-67 (prediluted, Ventana Medical Systems, Tucson, AZ), and CD34 (1:20, Abcam, Cambridge, MA) were performed on sections cut from the TMA blocks. Apoptotic cells were identified by terminal deoxynucleotidyl transferase-mediated dUTP nick end labeling (TUNEL) colorimetric staining (Promega, Madison, WI). Stained TMA slides were scanned (at 20x magnification setting) using the APERIO imaging system and the images were uploaded and viewed using TMAJ $[12,13]$. Each array spot was then formed into a composite image for viewing and scoring on a personal computer monitor.

2.5. Scoring of IHC Staining. IHC specimens were provided to pathologist for scoring. The identity of sample and treatment was blinded to the scorer. The specimens showed a varying degree of staining intensity and percentage of cells staining. Therefore, a combined intensity and percentage positive scoring method was used [14]. Strong intensity staining was scored as 3 , moderate as 2 , weak as 1 , and negative as 0 . For each intensity score, the percentage of cells with that score was estimated visually. A combined weighted score consisting of the sum of the percentage of cells staining at each intensity level was calculated for each sample, for example; a case with $50 \%$ strong staining, $20 \%$ moderate staining, and $10 \%$ weak staining would receive a score as follows: $(50 \times 3+20 \times 2+10 \times 1)=200$. The maximum score is 300 . The microvessel density (MVD) was calculated by counting all the vessels with the positive CD34 staining in the area of viable tissue.

2.6. Statistical Analysis. All statistical analyses were performed using GraphPad Prism version 4.0 (GraphPad Software Inc., San Diego, CA). Statistical significance was calculated using the Student's $t$-test to compare means. A value of $P<0.05$ was considered statistically significant.

\section{Results}

3.1. In Vivo Models. We have previously shown $40-70 \%$ reduction in tumor growth by in vivo treatment with $0.4 \%$ VPA in drinking water in LNCap, C4-2, and DU145 xenografts [7]. The present study focuses on effects on various pathways which may be behind the diminished growth of xenografts.

3.1.1. Chronic VPA in Drinking Water Causes Histone H3 Acetylation. In order to validate the effect of $0.4 \%$ VPA in drinking water on the xenografts, the acetylation status of histone $\mathrm{H} 3$ was assayed by IHC of the TMA. A significant increase in histone $\mathrm{H} 3$ acetylation was found in xenografts of all three cell lines of the VPA-treated animals when compared with the untreated controls (Figure 1(a) and Table 1).

3.1.2. Chronic VPA Treatment Causes Cell Cycle Arrest of Prostate Cancer Cells in Tumor Xenografts. The sequential and ordered activation of the cyclin-dependent kinases and inactivation of CDK inhibitors promote cell cycle progression. The accumulation of active cyclin D1-CDK4 complexes is 


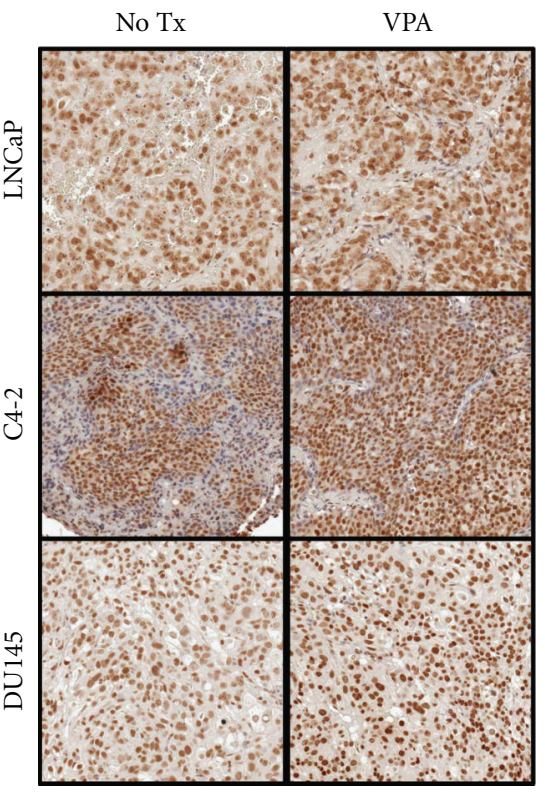

(a)

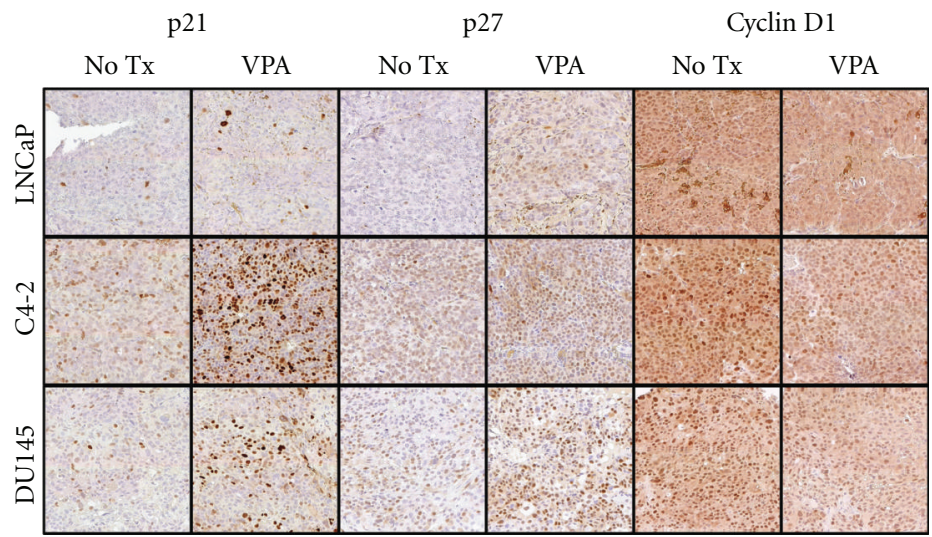

(b)

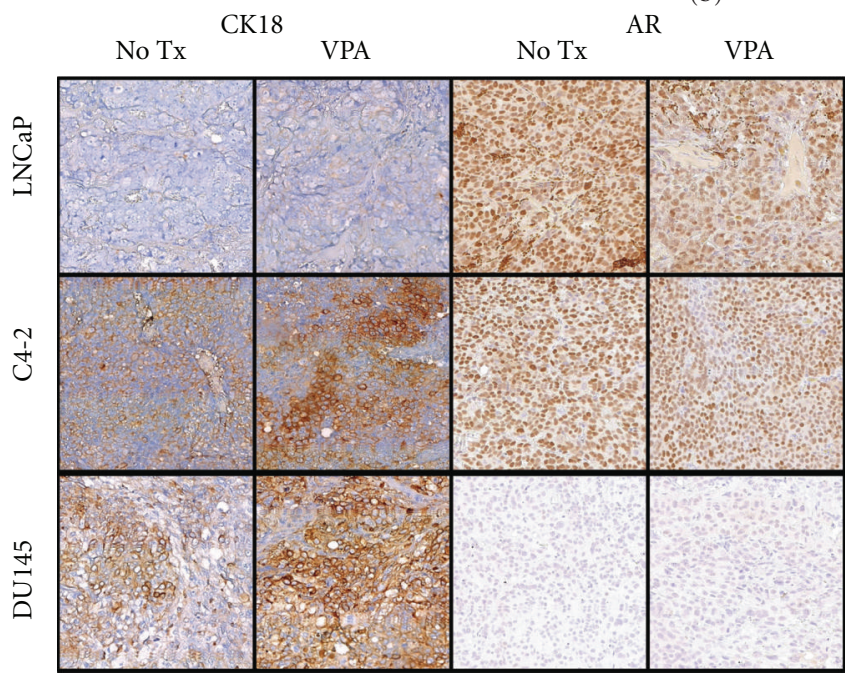

(c)

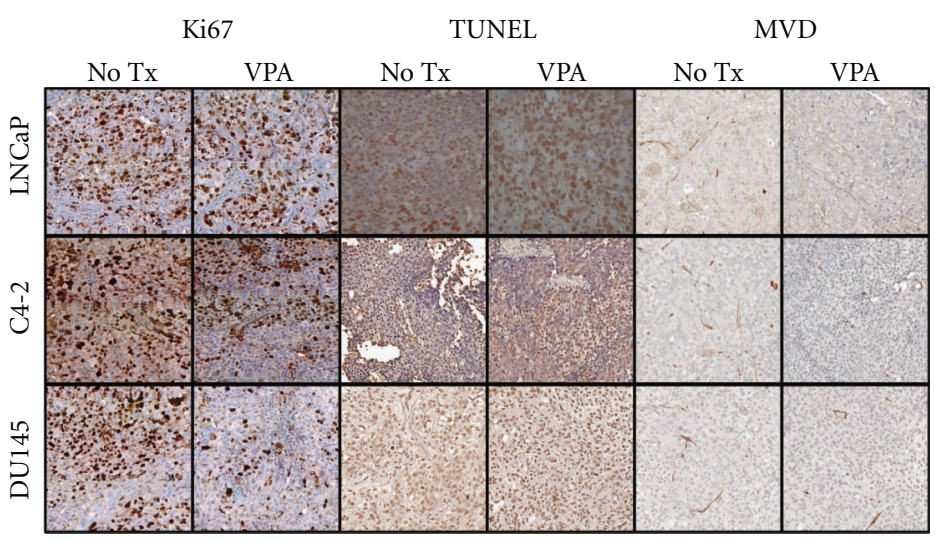

(d)

Figure 1: Animals with prostate cancer xenografts were randomized into control and treatment arms. Animals in treatment arm received $0.4 \% \mathrm{w} / \mathrm{v}$ Valproic Acid (VPA) in drinking water. Animals were treated for 35 days before excision of tumors. Tissue Microarrays (TMAs) of xenografts were created and expression of various markers was studied. Representative images of xenograft sections from control and VPA-treated groups; LNCaP, C4-2, and DU-145 (scanned at 20x magnification using the APERIO imaging system) are shown. (a) Immunohistochemistry (IHC) staining for acetylated histone 3. (b) Staining of cell cycle regulators (p21, p27, cyclin D1). (c) Staining for differentiation markers (Cytokeratin 18 (CK18), androgen receptor (AR)). (d) Staining for markers of proliferation (Ki-67), apoptosis (TUNEL), and angiogenesis (mean vascular density, MVD). 


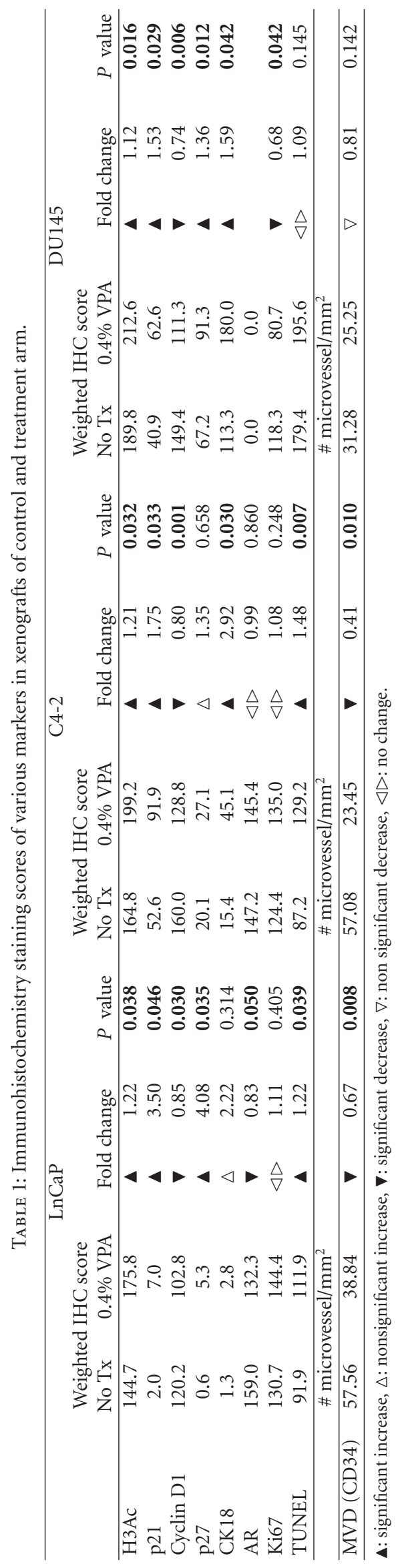


triggered by mitogenic stimuli through both increased cyclin expression and decreased cyclin proteolysis and through the promotion of cyclin D1-CDK4 assembly $[15,16]$. Some HDACIs have been previously shown to induce CDK inhibitor, $\mathrm{p} 21^{\mathrm{WAF} 1 / \mathrm{CIP} 1}$, in vitro. To study the effect in an in vivo model and to confirm that a pharmacologically sufficient dose of VPA reached the target tissue after oral administration, $\mathrm{p} 21^{\mathrm{WAF} 1 / \mathrm{CIP} 1}$ levels were measured in xenografts by TMA IHC. The xenografts in animals treated by VPA had significantly increased $\mathrm{p} 21^{\mathrm{WAF} 1 / \mathrm{CIP} 1}$ levels when compared to untreated controls (Figure 1(b) and Table 1). $\mathrm{p} 21^{\mathrm{WAF} 1 / \mathrm{CIP} 1}$ upregulation is an indication of cell cycle arrest and may account for the reduced tumor volumes of treated animals.

To further study the effects on cell cycle of VPA treatment, cyclin D1 and $\mathrm{p} 27^{\mathrm{KIP} 1}$ expression levels were quantified in the tumor TMA. Cyclin D1 levels decreased significantly in treated animals irrespective to tumor type (Figure 1(b) and Table 1), while p27 $7^{\mathrm{KIP} 1}$ levels increased significantly in LNCAP and DU145 xenografts but failed to achieve significance in C4-2 xenografts (Figure 1(b) and Table 1). These results further confirm the role of cell cycle gene regulation in the therapeutic effect of VPA and other HDACIs in prostate cancer.

\subsubsection{Chronic VPA Treatment Induces Differentiation Markers} in Cells. Certain classes of cytokeratins have also been used as differentiation markers for epithelial cells. Cytokeratin 18 (CK18) expression is associated with epithelial differentiation in many organs [17] including the prostate [18] and breast [19]. In this study we show CK18 expression increases in the VPA-treated C4-2 and DU145 xenografts (Figure 1(c) and Table 1) when compared to the untreated controls. The LNCaP xenografts show a similar increasing trend of CK18 expression. Thus VPA promotes epithelial differentiation to varying degrees in prostate cancer cells.

\subsubsection{Chronic VPA Treatment Decreases AR in LNCaP xen-} ografts. It has been suggested that it may be therapeutically beneficial to decrease the expression of the androgen receptor in prostate cancer [20]. We have previously shown that chronic VPA treatment of LNCaP cells in vitro results in reduction of AR expression [7]. Here we also find that LNCaP xenografts in animals treated with VPA in drinking water had reduced AR expression when compared to untreated animals (Figure 1(c) and Table 1). No change in AR expression was found in the androgen-independent C4-2 xenografts. The androgen-receptor-deficient DU145 xenografts as expected were negative for any AR expression irrespective of VPA treatment.

3.1.5. Mechanism of VPA-Induced Decrease in Tumor Growth Is Cell Type Dependent. Our previous study found that $0.4 \%$ VPA treatment causes a reduction in tumor growth [8]. In this study we have evaluated if this tumor reduction is due to decrease in proliferation or increase in apoptosis of tumor cells. Nuclear expression of the proliferation antigen Ki-67 has long been recognized to correlate with proliferating cells and tumor growth [21]. To discern the effects of chronic VPA treatment on proliferation, we looked at Ki-67 expression by IHC. Ki-67 expression did not significantly change in VPA-treated LNCaP and C4-2 xenografts (Figure 1(d) and Table 1), while that of DU145 decreased markedly. TUNEL assays were performed to quantify the degree of apoptosis in these models. VPA-treated LNCaP and C4-2 xenografts showed significantly increased apoptosis while DU145 xenografts had no change (Figure 1(d) and Table 1). Tumor reduction in DU145 xenografts were due to decreased proliferation while in LNCAP and C4-2 xenografts were due to increased apoptosis.

3.1.6. VPA Induces an Antiangiogenic Response. Angiogenesis is necessary for tumor growth and metastasis. The endothelial cells of blood vessels express CD34, which is commonly used as a marker for tumor neovasculature [22]. Micro vessel density (MVD) is a quantitative indication of the risk of tumor prognosis and metastases risk in various solid malignant tumors $[23,24]$. Here, MVD was evaluated by CD34 expression in the xenografts by TMA IHC. The number of vessels with positive CD34 staining per unit area $\left(\mathrm{mm}^{2}\right)$ of viable tissue was used to calculate MVD. The LNCaP and C4-2 xenografts showed a statistically significant decrease in MVD, from $57.56 \pm 6.64 / \mathrm{mm}^{2}$ and $57.08 \pm 8.21 / \mathrm{mm}^{2}$ to $38.96 \pm 5.17 / \mathrm{mm}^{2}$ and $23.45 \pm 3.21 / \mathrm{mm}^{2}$ respectively, following VPA treatment (Figure 1(d) and Table 1). The DU145 xenografts also showed a similar trend in reduction of MVD from $31.28 \pm 4.18 / \mathrm{mm}^{2}$ to $25.25 \pm 2.61 / \mathrm{mm}^{2}$ but failed to reach significance $(P=0.14)$.

3.2. In Vitro Models. The design of these studies included cell lines treated with various VPA doses as references and staining controls for the tumor xenograft TMA. LNCaP and C4-2 cells were treated with $0,0.6$ and $1.2 \mathrm{mM}$ VPA for 14 days in culture and harvested and incorporated as cell plugs into the TMA. As expected, the changes in p21, p27, and CK18 expression in vitro were similar to the effects of VPA on the xenografts. The $\mathrm{p} 21^{\mathrm{WAF} 1 / \mathrm{CIP} 1}$ and $\mathrm{p} 27^{\mathrm{KIP} 1}$ expression increased in a dose-dependent manner in both the LNCaP and C4-2 cells treated in vitro with VPA (Figure 2 and Table 2). At the same time CK18 levels also increased in a dose dependent manner in both cell lines tested (Figure 2 and Table 2). AR levels decreased in a dose-dependent manner in both LNCaP and C4-2 cells when treated with VPA in vitro (Figure 2 and Table 2). In contrast no change in AR expression was seen in C4-2 xenografts. While the proliferation of LNCaP and C4-2 cells in the xenografts was not affected by VPA treatment, the proliferative index as assessed by Ki-67 staining of both the cell types in vitro was significantly reduced in a dose-dependent manner (Figure 2 and Table 2). These results suggest that differential proliferative signaling pathways are present in the in vivo versus in vitro models.

\section{Discussion}

HDACIs represent a promising class of drugs for the alternative treatment of cancer because they have been proven to reactivate silenced tumor suppressor genes and have 
TABLE 2: Immunohistochemistry staining scores of various markers in tissue microarrays created from cell lines treated with $0,0.6$, and $1.2 \mathrm{mM}$ of valproic acid for 14 days.

\begin{tabular}{|c|c|c|c|c|c|c|c|c|c|c|}
\hline \multirow{4}{*}{$\frac{\text { VPA }}{\text { p21 }}$} & \multicolumn{10}{|c|}{$\mathrm{LnCaP}$ (in vitro) } \\
\hline & \multicolumn{3}{|c|}{ Weighted IHC score } & \multicolumn{4}{|c|}{ Fold change } & \multicolumn{3}{|c|}{$P$ value } \\
\hline & $0 \mathrm{mM}$ & $0.6 \mathrm{mM}$ & $1.2 \mathrm{mM}$ & \multicolumn{2}{|c|}{$0.6 \mathrm{mM}$} & \multicolumn{2}{|c|}{$1.2 \mathrm{mM}$} & 0 versus 0.6 & 0 versus 1.2 & 0.6 versus 1.2 \\
\hline & 50 & 65 & 110 & $\Delta$ & 1.30 & $\Delta$ & 2.20 & $<0.001$ & $<0.001$ & $<0.001$ \\
\hline $\mathrm{p} 27$ & 7 & 13 & 17 & $\Delta$ & 1.86 & $\Delta$ & 2.43 & $<0.001$ & $<0.001$ & $<0.001$ \\
\hline CK18 & 36 & 53 & 115 & $\Delta$ & 1.47 & $\Delta$ & 3.19 & $<0.001$ & $<0.001$ & $<0.001$ \\
\hline $\mathrm{AR}$ & 164 & 150 & 117 & $\nabla$ & 0.91 & $\nabla$ & 0.71 & $<0.001$ & $<0.001$ & $<0.001$ \\
\hline \multirow[t]{3}{*}{ Ki67 } & 230 & 175 & 135 & $\nabla$ & 0.76 & $\nabla$ & 0.59 & $<0.001$ & $<0.001$ & $<0.001$ \\
\hline & \multicolumn{10}{|c|}{ C4-2 (in vitro) } \\
\hline & \multicolumn{3}{|c|}{ Weighted IHC score } & \multicolumn{4}{|c|}{ Fold change } & \multicolumn{3}{|c|}{$P$ value } \\
\hline VPA & $0 \mathrm{mM}$ & $0.6 \mathrm{mM}$ & $1.2 \mathrm{mM}$ & & $\mathrm{M}$ & & & 0 versus 0.6 & 0 versus 1.2 & 0.6 versus 1.2 \\
\hline $\mathrm{p} 21$ & 85 & 100 & 140 & $\Delta$ & 1.18 & $\boldsymbol{\Delta}$ & 1.65 & $<0.001$ & $<0.001$ & $<0.001$ \\
\hline p27 & 42 & 58 & 73 & $\Delta$ & 1.38 & $\Delta$ & 1.74 & $<0.001$ & $<0.001$ & $<0.001$ \\
\hline CK18 & 65 & 80 & 105 & $\Delta$ & 1.23 & $\Delta$ & 1.62 & $<0.001$ & $<0.001$ & $<0.001$ \\
\hline $\mathrm{AR}$ & 220 & 200 & 185 & $\nabla$ & 0.91 & $\nabla$ & 0.84 & $<0.001$ & $<0.001$ & $<0.001$ \\
\hline Ki67 & 200 & 180 & 100 & $\nabla$ & 0.90 & $\nabla$ & 0.50 & $<0.001$ & $<0.001$ & $<0.001$ \\
\hline
\end{tabular}

$\mathbf{\Delta}$ : significant increase, $\triangle$ : nonsignificant increase, $\mathbf{\nabla}$ : significant decrease, $\nabla$ : non significant decrease, $\triangleleft \triangleright$ : no change.

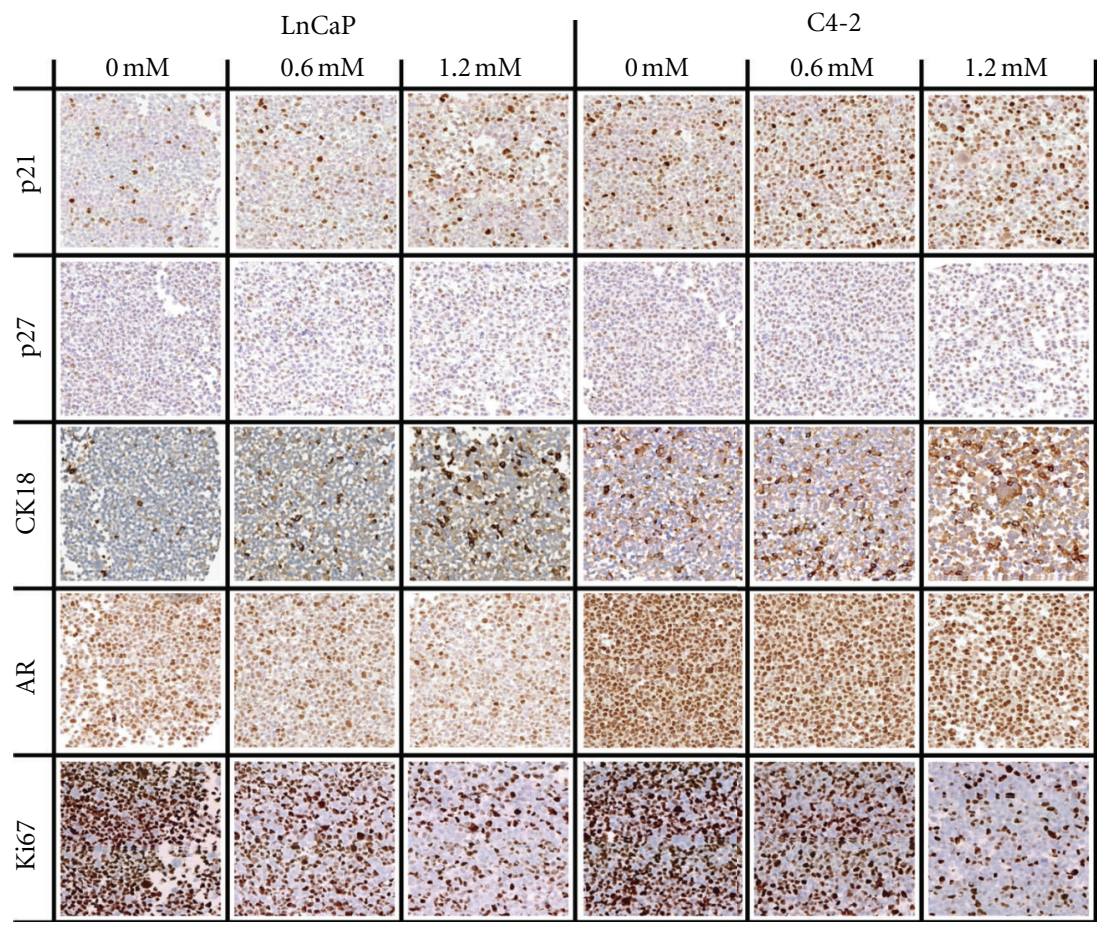

FIGURE 2: Cell lines (LNCaP, C4-2) were treated with 0, 0.6, and $1.2 \mathrm{mM}$ of VPA for 14 days. TMA were created from cell pellets followed by IHC staining for expression of markers. Representative images of p21, p27, CK18, AR, and Ki-67 staining are shown (scanned at 20x magnification using the APERIO imaging system).

pleiotropic antitumor effects selectively in cancer cells [25]. This is particularly appealing because most human cancers are highly heterogeneous and potentially involve many different signaling pathways. HDACIs have shown significant activity against a variety of hematologic and solid tumor model systems both in vitro and in vivo [26-28]. Our previous study found that chronic administration of VPA results in a marked decrease in proliferation of prostate cancer cells in vitro and significant reduction in tumor volume in vivo [7]. In order to further explore the mechanism by 
which VPA inhibited xenograft growth, we generated a tissue microarray (TMA) from treated and untreated xenograft tumors and analyzed the TMA for expression of cell-cycleassociated markers and proliferation markers.

The HDACI activity of VPA was confirmed by the evaluation of a dose-dependent increase in both acetylated $\mathrm{H} 3$ and p2 $1^{\mathrm{WAF} 1 / \mathrm{CIP} 1}$ expression in VPA-treated LNCaP, C4-2 and DU145 xenografts. This result is consistent with our previous studies showing that both acute and chronic VPA treatment can increase net histone $\mathrm{H} 3$ acetylation and upregulate p $21^{\mathrm{WAF} / \mathrm{CIP} 1}$ expression $[7,8]$. Xia et al. demonstrated the upregulation of p21 by western blotting. Our current study has demonstrated similar results by IHC further validating the results. $21^{\mathrm{WAFI} / \mathrm{CIP} 1}$ hampers cell cycle progression by inhibiting the activity of cyclin/CDK2 complexes and blocks DNA replication by binding to the proliferating cell nuclear antigen. The induction of $\mathrm{p} 21^{\mathrm{WAF} 1 / \mathrm{CIP} 1}$ expression is important for the potential therapeutic effects of HDACI [29]. Also, p2 $7^{\mathrm{KIPl}}$ has been demonstrated to play an important role in arresting the progression from G1 to S phase of the cell cycle by preventing activation of cyclin E/CDK2 complexes [30]. Our data indicates that after VPA treatment, $\mathrm{p} 27^{\mathrm{KIP} 1}$ also showed an increased expression both in vitro and in vivo.

Cyclin D1 is an important positive regulator of the G1-S phase cell cycle transition. As the best characterized member of the D-type cyclin family, cyclin D1 binds to and activates CDK4/6 complexes, contributing to the phosphorylation and inactivation of retinoblastoma tumor suppressor gene protein, resulting in cell cycle progression $[31,32]$. Cyclin D1 becomes amplified or overexpressed in many different tumor types thus promoting tumor growth. Our results show that expression of cyclin D1 was decreased in LNCaP, C42 , and DU145 in vivo via VPA treatment, which may have contributed to the inhibition of tumor growth.

Loss of cytokeratin expression has been shown to be a poor prognostic factor for patients with breast cancer [33]. Studies show that cell death in VPA-treated cells may occur by the transcriptional changes in genes that are involved in differentiation and apoptosis [34]. Our results show that the expression of CK18 was increased in VPA-treated C42 and DU145 xenografts, which may indicate that VPA can also elicit cellular differentiation to inhibit the growth of PCa cell lines in vivo. Furthermore, it has now been demonstrated that $\mathrm{AR}$ and its signaling axis remain involved in the survival and growth of prostate cancer cells, despite the depletion of circulating androgenic ligands. AR amplification with concomitant overexpression of $\mathrm{AR}$ can increase the sensitivity of prostate cancer cells to low levels of androgens that eventually result in the development of androgenindependent prostate cancer [35]. Therefore, there has been much interest in developing therapies functioning partly by downregulating AR expression in prostate tumors. Our previous report shows that chronic treatment with VPA led to a net decrease in AR protein in $\mathrm{LNCaP}$ cell lines in vitro (shown by western blotting) [7]. This has been reproduced in vivo in the present study by using IHC. This result further establishes the comparability of IHC to western blotting in this scenario. In case of C4-2 xenografts, no change in AR expression was seen after VPA treatment, while AR expression decreased in vitro. The difference could be the result of different behavior of cells in vivo when compared to in vitro, particularly given the different microenvironment.

Primary mechanisms of HDACIs' anticancer effects lie in the inhibition of cell growth and induction of apoptosis. As a proliferation marker, Ki-67 expression was significantly decreased in a dose-dependent manner in LNCaP, C4-2 cell lines in vitro upon VPA treatment, while the same decreasing expression of Ki67 was only observed in androgenindependent DU145 VPA-treated xenografts. So chronic VPA treatment inhibits proliferation of cells in androgenindependent tumors of DU145 but lacks similar effect on androgen-dependent tumors of LNCaP and C4-2. The differences between in vivo and in vitro observations may be attributable to the pharmacology of VPA; the production, distribution, and metabolism of VPA in vivo may result in different bioavailable doses and duration of VPA reaching the target tumor site than can be achieved in vitro. These results show the importance of validating in vitro data in an in vivo model, as in vitro data does not always recapitulate in an in vivo model. Our data also show VPA causes cell death of LNCaP and C4-2 cells in vivo by increasing the rate of apoptosis, although there is no difference of $\mathrm{Ki}-67$ expression in the latter two cell lines in vivo. Thus, inhibition of proliferation and induction of apoptosis may be two independent events. Previously in the manuscript by Shabbeer et al. [8] induction of apoptosis was demonstrated in DU145 xenografts by VPA treatment, while in our present study no significant change in apoptosis was seen. We checked serum levels of VPA in animals and we found that serum levels varied widely depending on the timing of ingestion of water with VPA, as the mice metabolized VPA much faster than humans (20 min half-life versus 16 hrs in humans). In vitro, DU145 seemed to exhibit a very sharp induction curve for apoptosis with relative resistance until certain levels (typically $2.5 \mathrm{mM}$ ) were reached after which the induction of apoptosis was profound (data not shown). Other cell lines had a more linear relationship between dose and effect. In our analysis we presented aggregate data of all animals, where serum VPA levels may not have peaked as sharply as the experiment presented in manuscript of Shabbeer et al. Since our experiments were an aggregate of all the animals, we believe it to be more representative.

HDACIs cause antiangiogenic effects by inhibiting proangiogenic and activating antiangiogenic gene and/or protein expression in cancer cells. HDACIs can also act on endothelial cells directly by downregulating the expression of survivin, an inhibitor of apoptosis in proliferating endothelial cells [24]. MVD is a prognostic factor and an independent predictor of pathological stages and malignant potential of prostate cancer [36]. CD34 expression was found to be associated with endothelial microprocesses occurring at the tips of vascular sprouts in the tumor stroma, where angiogenesis was progressing [37]. Several studies indicate that anti-CD34 antibody is superior to several other antibodies for microvessel counting and has a better relation to patient prognosis $[23,38]$. Our data show that VPA treatment triggered an antiangiogenic effect in terms of a less dense vasculature in LNCaP and C4-2. This inhibition of 
angiogenesis may also be a contributing factor for decreased tumor growth in VPA-treated animals.

In this study we have shown that cell cycle arrest, differentiation, and inhibition of tumor vasculature, all play a role in the growth inhibition of PCa cells treated with VPA. VPA induces cell cycle arrest in PCa cells in vivo, as seen by the increase in $\mathrm{p} 21^{\mathrm{WAF} 1 / \mathrm{CIP} 1}$ and decrease in cyclin D1 expression. VPA-treated xenografts show an increase in differentiation markers such as p27 and CK18. While decrease in proliferation was observed in vitro, apoptosis was the primary cause of diminished tumor growth in vivo in AR-positive LNCap and C4-2 xenografts. Chronic VPA treatment also results in an antiangiogenic effect on $\mathrm{PCa}$ cells. To conclude, VPA-induced inhibition of tumor growth occurs through multiple mechanisms.

\section{Nonstandard Abbreviations}

$\begin{array}{ll}\text { VPA: } & \text { Valproic acid } \\ \text { PCa: } & \text { Prostate cancer } \\ \text { HDAC: } & \text { Histone deacetylase } \\ \text { HDACI: } & \text { Histone deacetylase inhibitor } \\ \text { TMA: } & \text { Tissue microarray } \\ \text { IHC: } & \text { Immunohistochemistry } \\ \text { CK18: } & \text { Cytokeratin } 1 \\ \text { CDKI: } & \text { Cyclin-dependent kinase inhibitor } \\ \text { AR: } & \text { Androgen receptor } \\ \text { TUNEL: } & \text { Terminal deoxynucleotidyl } \\ & \text { transferase-mediated dUTP nick end labeling } \\ \text { MVD: } & \text { Mean vascular density. }\end{array}$

\section{Authors' Contribution}

A. Sidana and M. Wang have contributed equally to the work.

\section{Acknowledgment}

This work was performed by a philanthropic support of Mr. Harvey Clapp.

\section{References}

[1] A. Rephaeli, D. Blank-Porat, N. Tarasenko et al., "In vivo and in vitro antitumor activity of butyroyloxymethyl-diethyl phosphate (AN-7), a histone deacetylase inhibitor, in human prostate cancer," International Journal of Cancer, vol. 116, no. 2, pp. 226-235, 2005.

[2] P. A. Konstantinopoulos, M. V. Karamouzis, and A. G. Papavassiliou, "Focus on acetylation: the role of histone deacetylase inhibitors in cancer therapy and beyond," Expert Opinion on Investigational Drugs, vol. 16, no. 5, pp. 569-571, 2007.

[3] M. Dokmanovic and P. A. Marks, "Prospects: histone deacetylase inhibitors," Journal of Cellular Biochemistry, vol. 96, no. 2, pp. 293-304, 2005.

[4] Y. Sowa, T. Orita, S. Hiranabe-Minamikawa et al., "Histone deacetylase inhibitor activates the p21/WAF1/Cip1 gene promoter through the Sp1 sites," Annals of the New York Academy of Sciences, vol. 886, pp. 195-199, 1999.
[5] M. V. Blagosklonny, "Are p27 and p21 cytoplasmic oncoproteins?" Cell Cycle (Georgetown, Tex.), vol. 1, no. 6, pp. 391-393, 2002.

[6] M. Göttlicher, S. Minucci, P. Zhu et al., "Valproic acid defines a novel class of HDAC inhibitors inducing differentiation of transformed cells," The EMBO Journal, vol. 20, no. 24, pp. 6969-6978, 2002.

[7] Q. Xia, J. Sung, W. Chowdhury et al., "Chronic administration of valproic acid inhibits prostate cancer cell growth in vitro and in vivo," Cancer Research, vol. 66, no. 14, pp. 7237-7244, 2006.

[8] S. Shabbeer, M. S. Q. Kortenhorst, S. Kachhap, N. Galloway, R. Rodriguez, and M. A. Carducci, "Multiple molecular pathways explain the anti-proliferative effect of valproic acid on prostate cancer cells in vitro and in vivo," Prostate, vol. 67, no. 10, pp. 1099-1110, 2007.

[9] C.-L. Chen, J. Sung, M. Cohen et al., "Valproic acid inhibits invasiveness in bladder cancer but not in prostate cancer cells," Journal of Pharmacology and Experimental Therapeutics, vol. 319, no. 2, pp. 533-542, 2006.

[10] D. M. Turnbull, M. D. Rawlins, D. Weightman, and D. W. Chadwick, "Plasma concentrations of sodium valproate: their clinical value," Annals of Neurology, vol. 14, no. 1, pp. 38-42, 1983.

[11] S. Zha, W. R. Gage, J. Sauvageot et al., "Cyclooxygenase-2 is up-regulated in proliferative inflammatory atrophy of the prostate, but not in prostate carcinoma," Cancer Research, vol. 61, no. 24, pp. 8617-8623, 2001.

[12] S. Manley, N. R. Mucci, A. M. De Marzo, and M. A. Rubin, "Relational database structure to manage high-density tissue microarray data and images for pathology studies focusing on clinical outcome the prostate specialized program of research excellence model," American Journal of Pathology, vol. 159, no. 3, pp. 837-843, 2001.

[13] D. A. Faith, W. B. Isaacs, J. D. Morgan et al., "Trefoil factor 3 overexpression in prostatic carcinoma: prognostic importance using tissue microarrays," Prostate, vol. 61, no. 3, pp. 215-227, 2004.

[14] A. M. De Marzo, B. Knudsen, K. Chan-Tack, and J. I. Epstein, "E-cadherin expression as a marker of tumor aggressiveness in routinely processed radical prostatectomy specimens," Urology, vol. 53, no. 4, pp. 707-713, 1999.

[15] C. Marshall, "How do small GTPase signal transduction pathways regulate cell cycle entry?" Current Opinion in Cell Biology, vol. 11, no. 6, pp. 732-736, 1999.

[16] J. R. Alt, A. B. Gladden, and J. A. Diehl, "p21Cip1 promotes cyclin D1 nuclear accumulation via direct inhibition of nuclear export," The Journal of Biological Chemistry, vol. 277, no. 10, pp. 8517-8523, 2002.

[17] W. K. Schlage, H. Bülles, D. Friedrichs, M. Kuhn, A. Teredesai, and P. M. Terpstra, "Cytokeratin expression patterns in the rat respiratory tract as markers of epithelial differentiation in inhalation toxicology. II. Changes in cytokeratin expression patterns following 8-day exposure to room-aged cigarette sidestream smoke," Toxicologic Pathology, vol. 26, no. 3, pp. 344-360, 1998.

[18] Y. Wang, S. W. Hayward, M. Cao, K. A. Thayer, and G. R. Cunha, "Cell differentiation lineage in the prostate," Differentiation, vol. 68, no. 4-5, pp. 270-279, 2001.

[19] G. Schaller, I. Fuchs, W. Pritze et al., "Elevated keratin 18 protein expression indicates a favorable prognosis in patients with breast cancer," Clinical Cancer Research, vol. 2, no. 11, pp. 1879-1885, 1996. 
[20] A. F. Santos, H. Huang, and D. J. Tindall, "The androgen receptor: a potential target for therapy of prostate cancer," Steroids, vol. 69, no. 2, pp. 79-85, 2004.

[21] V. Ananthanarayanan, R. J. Deaton, X. J. Yang, M. R. Pins, and P. H. Gann, "Alteration of proliferation and apoptotic markers in normal and premalignant tissue associated with prostate cancer," BMC Cancer, vol. 6, article 73, 2006.

[22] L. Fina, H. V. Molgaard, D. Robertson et al., "Expression of the CD34 gene in vascular endothelial cells," Blood, vol. 75, no. 12, pp. 2417-2426, 1990.

[23] E. Yildiz, S. Ayan, F. Goze, G. Gokce, and E. Y. Gultekin, "Relation of microvessel density with microvascular invasion, metastasis and prognosis in renal cell carcinoma," BJU International, vol. 101, no. 6, pp. 758-764, 2008.

[24] T. Liu, S. Kuljaca, A. Tee, and G. M. Marshall, "Histone deacetylase inhibitors: multifunctional anticancer agents," Cancer Treatment Reviews, vol. 32, no. 3, pp. 157-165, 2006.

[25] R. R. Rosato and S. Grant, "Histone deacetylase inhibitors: insights into mechanisms of lethality," Expert Opinion on Therapeutic Targets, vol. 9, no. 4, pp. 809-824, 2005.

[26] L. C. Sambucetti, D. D. Fischer, S. Zabludoff et al., "Histone deacetylase inhibition selectively alters the activity and expression of cell cycle proteins leading to specific chromatin acetylation and antiproliferative effects," The Journal of Biological Chemistry, vol. 274, no. 49, pp. 34940-34947, 1999.

[27] S. Iwata, T. Saito, Y. Ito et al., "Antitumor activities of valproic acid on Epstein-Barr virus-associated T and natural killer lymphoma cells," Cancer Science, vol. 103, no. 2, pp. 375-381, 2012.

[28] A. Kuendgen and N. Gattermann, "Valproic acid for the treatment of myeloid malignancies," Cancer, vol. 110, no. 5, pp. 943-954, 2007.

[29] M. Ocker and R. Schneider-Stock, "Histone deacetylase inhibitors: signalling towards p21cip1/waf1," International Journal of Biochemistry and Cell Biology, vol. 39, no. 7-8, pp. 1367-1374, 2007.

[30] W. Li, A. Sanki, R. Z. Karim et al., "The role of cell cycle regulatory proteins in the pathogenesis of melanoma," Pathology, vol. 38, no. 4, pp. 287-301, 2006.

[31] J. Bartek, J. Bartkova, and J. Lukas, "The retinoblastoma protein pathway and the restriction point," Current Opinion in Cell Biology, vol. 8, no. 6, pp. 805-814, 1996.

[32] D. Parry, D. Mahony, K. Wills, and E. Lees, "Cyclin DCDK subunit arrangement is dependent on the availability of competing INK4 and p21 class inhibitors," Molecular and Cellular Biology, vol. 19, no. 3, pp. 1775-1783, 1999.

[33] B. Willipinski-Stapelfeldt, S. Riethdorf, V. Assmann et al., "Changes in cytoskeletal protein composition indicative of an epithelial-mesenchymal transition in human micrometastatic and primary breast carcinoma cells," Clinical Cancer Research, vol. 11, no. 22, pp. 8006-8014, 2005.

[34] P. A. Marks, V. M. Richon, and R. A. Rifkind, "Histone deacetylase inhibitors: inducers of differentiation or apoptosis of transformed cells," Journal of the National Cancer Institute, vol. 92, no. 15, pp. 1210-1216, 2000.

[35] J. T. Isaacs and W. B. Isaacs, "Androgen receptor outwits prostate cancer drugs," Nature Medicine, vol. 10, no. 1, pp. 26-27, 2004.

[36] I. U. Ali, D. R. Senger, and L. E. H. Smith, "Angiogenesis as a potential biomarker in prostate cancer chemoprevention trials," Urology, vol. 57, no. 4, pp. 143-147, 2001.

[37] R. O. Schlingemann, F. J. R. Rietveld, R. M. W. De Waal et al., "Leukocyte antigen CD34 is expressed by a subset of cultured endothelial cells and on endothelial abluminal microprocesses in the tumor stroma," Laboratory Investigation, vol. 62, no. 6, pp. 690-696, 1990.

[38] E. Sabo, A. Boltenko, Y. Sova, A. Stein, S. Kleinhaus, and M. B. Resnick, "Microscopic analysis and significance of vascular architectural complexity in renal cell carcinoma," Clinical Cancer Research, vol. 7, no. 3, pp. 533-537, 2001. 


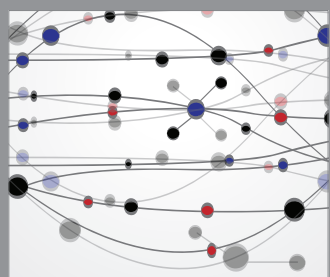

The Scientific World Journal
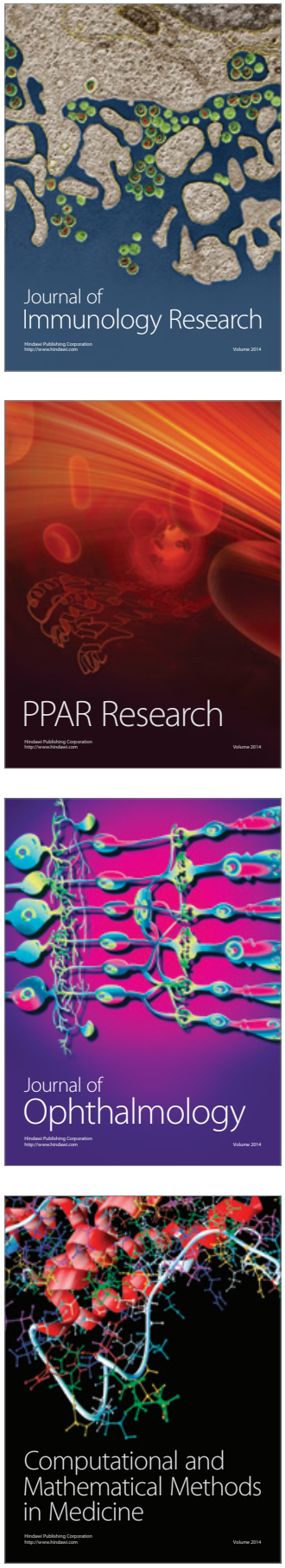

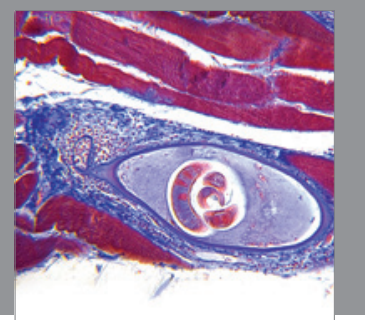

Gastroenterology

Research and Practice
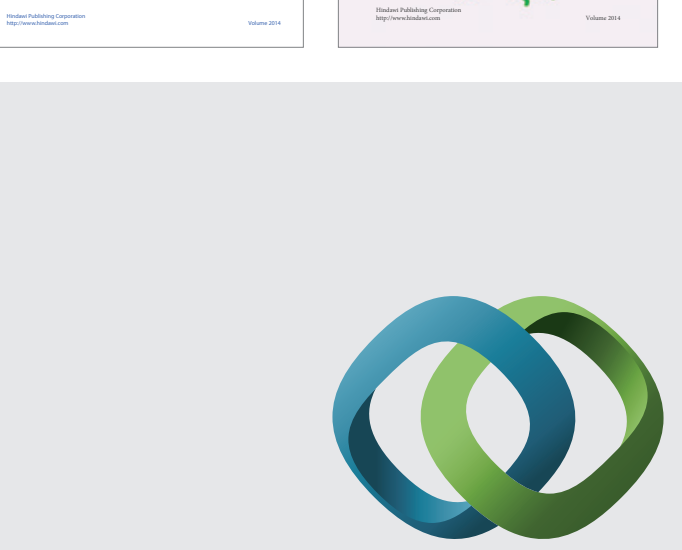

\section{Hindawi}

Submit your manuscripts at

http://www.hindawi.com
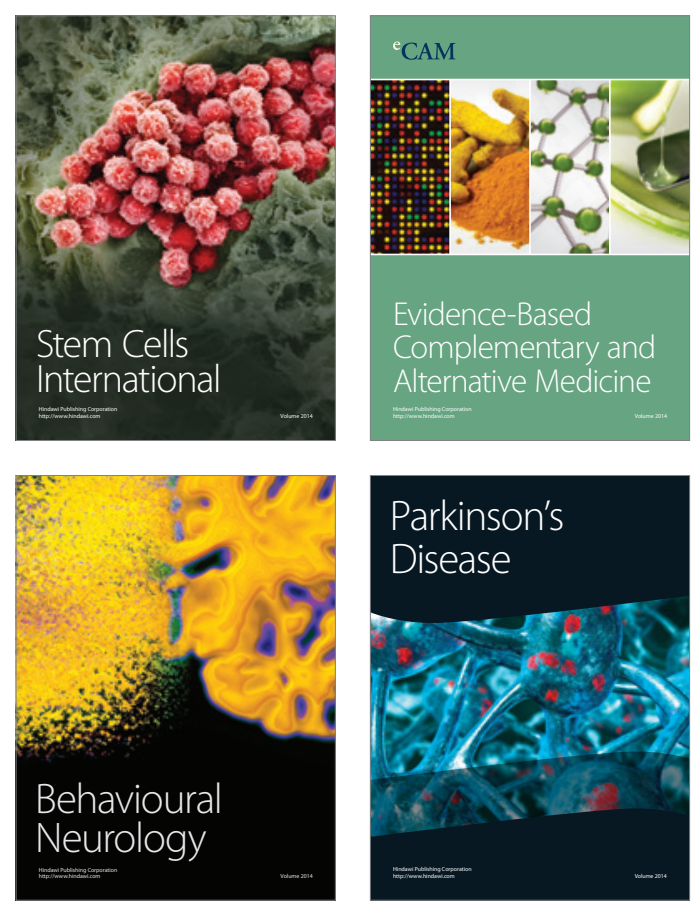

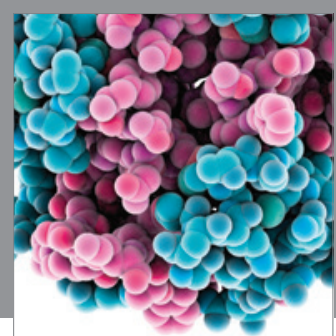

Journal of
Diabetes Research

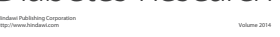

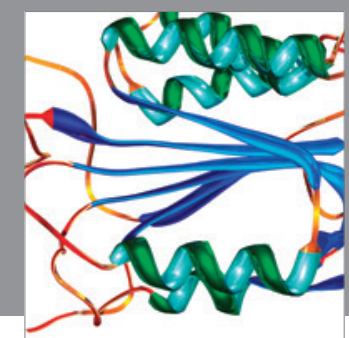

Disease Markers
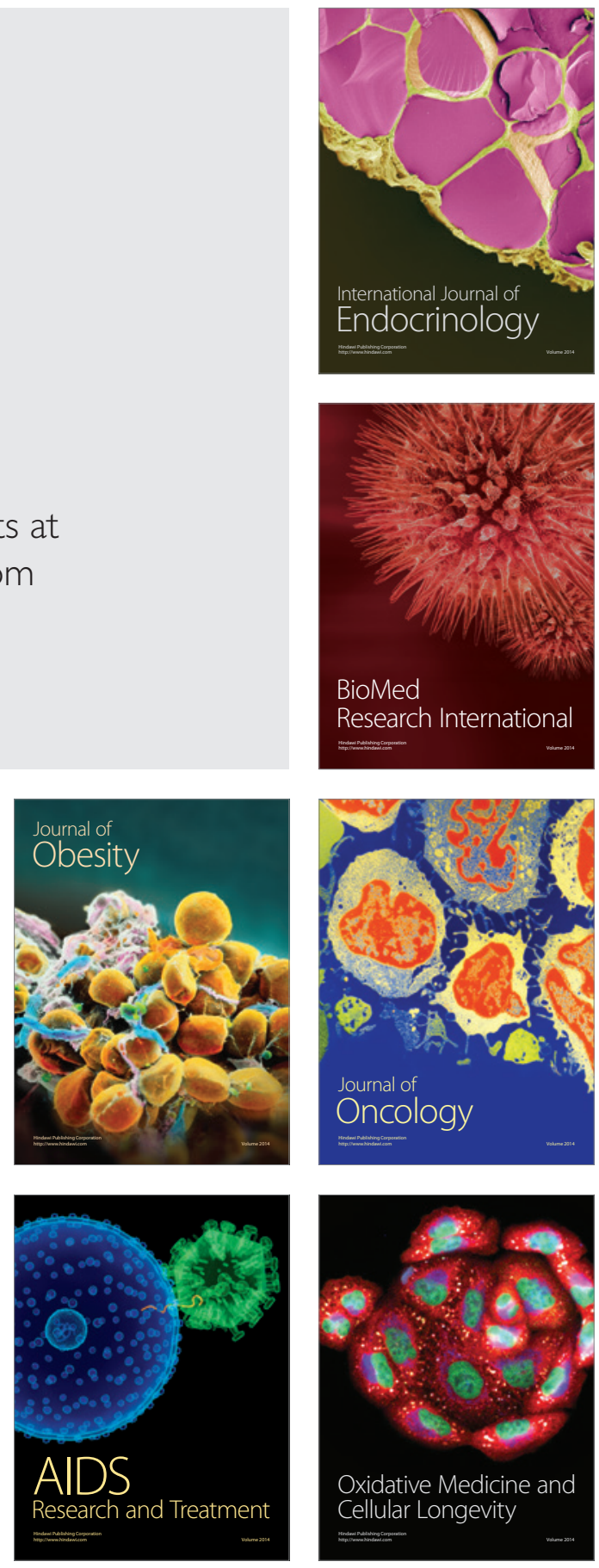